\title{
Image Quality assessment for Awake Animal Brain PET
}

\author{
Simone Beer, Alan Miranda, David Elmenhorst, Tina Kroll, Jeroen Verhaeghe and Andreas Bauer
}

\begin{abstract}
A restrictive issue for small-animal positron emission tomography (PET) imaging is the fact that animals are either anaesthetized or physically restrained to prevent them from moving during the imaging procedure. Tracking the motion of the animal, based on e.g. radioactive point sources which are attached to the head of the animal, allows to scan awake animals. The use of radioactive point sources for motion tracking has the benefit that it can be used in scanners with small bore size or occluded views, is minimally invasive and does not require additional precise calibration between the PET camera and an external tracking device. The aim of this study was to assess the image quality and quantitative accuracy for PET scans of moving objects, with motion correction based on radioactive point sources, following the procedures of the NEMA NU 4-2008 standard. We found that even extensive motion of an object, including rotation and turning front to back, affects image quality as well as quantitation only marginally compared to the motion free scan.
\end{abstract}

\section{INTRODUCTION}

SMALL-ANimal PET with mice and rats is a powerful tool to $S_{\text {study e.g. etiologies, progression of disease and novel }}$ treatment strategies. However, a restrictive issue for smallanimal imaging is the fact that animals have to be either anaesthetized or physically restrained to prevent them from moving during the imaging procedure, which would result in unacceptable blurring of the images. When imaging the brain with PET, both anaesthesia and stress caused by physical restraints have a high chance to alter the neurochemistry which is supposed to be measured. For example, DeLorenzo et al [1] have shown that the anaesthetic agent ketamine might decrease the binding of $\left[{ }^{11} \mathrm{C}\right] \mathrm{ABP} 688$, an mGluR5 receptor ligand.

Alstrup and Smith [2] have reviewed effects of different anaesthesia on the outcome of PET receptor imaging in animals. Depending on the PET tracer, different anaesthetics cause either an increase or a decrease in ligand binding. They note that the effects of anaesthetics on brain mechanisms are species-specific and dose-dependent.

Manuscript received December 9, 2019. This work was part of the ERANET NEURON project SleepLess supported by BMBF (01EW1808), FWO and FRQS under the frame of Neuron Cofund.

Simone Beer is with the Institute of Neuroscience and Medicine (INM-2), Forschungszentrum Jülich, Jülich, Germany (telephone: +49-2461-611954, email: si.beer@fz-juelich.de).

Alan Miranda and Jeroen Verhaeghe are with the Molecular Imaging Center Antwerp, University of Antwerp, Belgium.

David Elmenhorst, Tina Kroll and Andreas Bauer are with the Institute of Neuroscience and Medicine (INM-2), Forschungszentrum Jülich, Jülich, Germany.
It has previously been proposed to perform PET imaging of unrestrained awake animals [3] to eliminate the detrimental effects of anaesthesia and restraining on the PET reading. The idea of most approaches relies on tracking the animal's head during scanning and then to take this additional information into account during PET image reconstruction, resulting in an image as if no motion had occurred.

Several methods have been used to track the motion of a rat within preclinical PET scanners, either with markers attached to the rat head and optical motion tracking [4], with markerless motion tracking relying on natural features of the rat head [5], or based on radioactive point sources attached to the rat head [6]. The use of radioactive point sources for motion tracking has the benefit that it can be used in scanners with small bore size or occluded views, is minimally invasive and does not require additional precise calibration between the PET camera and an external tracking device. All these factors contribute to the practicality of the motion tracking method, opening the way to routine use in the preclinical PET imaging lab. Neuroreceptor PET studies are commonly analyzed by applying tracer kinetic models to the PET time-activity data. The outcome measure is usually the distribution volume (VT) or the binding potential (BP), which reflect the densities of target proteins in a brain region of interest. The application of a tracer kinetic model requires quantitative PET image data. The aim of this study was therefore to assess the image quality and quantitative accuracy for PET scans of moving objects, with motion correction based on radioactive point sources, following the procedures of the NEMA NU 4-2008 standard [7].

\section{MATERIAL AND METHODS}

\section{A. NEMA NU 4 Image Quality Phantom}

The NEMA NU 4-2008 image quality phantom is a $50 \mathrm{~mm}$ long, $30 \mathrm{~mm}$ diameter PMMA cylinder which consists of 1) a fillable cylindrical chamber with $30 \mathrm{~mm}$ diameter and $30 \mathrm{~mm}$ length; 2) a $20 \mathrm{~mm}$ long solid chamber with 5 fillable rods with diameters of $1,2,3,4$, and $5 \mathrm{~mm}$, respectively; 3 ) a lid attached to the large uniform region supporting two cold region chambers, $15 \mathrm{~mm}$ in length and $8 \mathrm{~mm}$ diameter, one filled with non-radioactive water and one filled with air.

\section{B. Data Acquisition and Image Reconstruction}

The phantom was filled with $3.57 \mathrm{MBq}(96.5 \mu \mathrm{Ci}){ }^{18} \mathrm{~F}$ at the time of the start of the first emission scan, which corresponds to an activity concentration of $163.5 \mathrm{kBq} / \mathrm{cc}$. It was first 
scanned for $20 \mathrm{~min}$ in a static position, after a $10 \mathrm{~min}$ transmission scan, in a Siemens Inveon small-animal PET scanner. Then, three point sources $(\sim 260 \mathrm{kBq}$ each, superabsorbent polymer, $<1 \mathrm{~mm} \varnothing$ (Figure 1)) were attached to the surface of the cylinder, and the phantom was scanned for another $20 \mathrm{~min}$, with extensive motion including rotation and turning front to back. The phantom was also moved temporarily to axial positions far away from the center of the field-of-view (FOV), where the sensitivity of the scanner is very low. The activity concentration in the phantom at the time of the motion scan was $128 \mathrm{kBq} / \mathrm{cc}$.

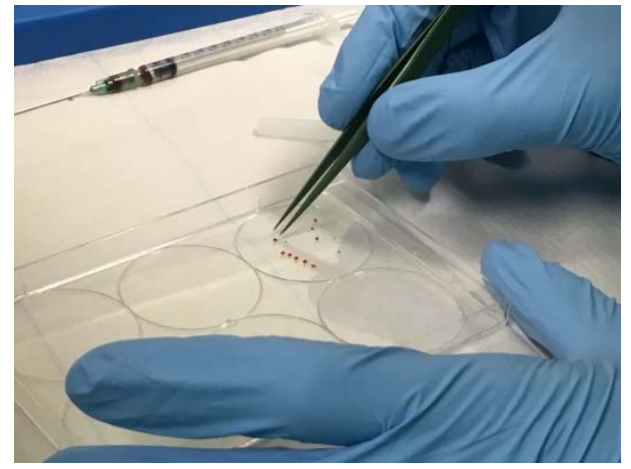

Fig. 1. Point sources. Colored for better visualization, soaked with radioactive liquid.

The static data were reconstructed with software provided by the vendor (Siemens Inveon IAW 2.1), using OSEM3DSP-MAP with 2 OSEM iterations, 18 MAP iterations and 1.5 $\mathrm{mm}$ target resolution. Both attenuation and scatter correction, based on the transmission scan, were applied.

The motion data were reconstructed using ML-EM with 8 iterations and 16 subsets, with point source motion tracking and image-space based resolution modelling [8] using a Gaussian with a full-width-at-half-maximum (FWHM) of 1.6 $\mathrm{mm}$. Attenuation correction was applied using an attenuation map calculated from the activity contour of the phantom [9]. Scatter correction was performed using the motion corrected sinograms and the single scatter simulation.

\section{Image Analysis}

Reconstructed images were analyzed using PMOD (version 3.408, PMOD Group, Zurich, Switzerland), following the procedures provided by the NEMA NU 4-2008 standard [7]. A $22.5 \mathrm{~mm}$ diameter by $10 \mathrm{~mm}$ long volume-of-interest (VOI) was drawn over the center of the uniform region, and the average activity concentration as well as the $\% \mathrm{SD}$ as a measure of uniformity was determined. The average activity concentrations of two VOIs of $4 \mathrm{~mm}$ diameter and $7.5 \mathrm{~mm}$ length, centered in the water- and air-filled inserts, were related to the average activity concentration of the uniform region as spill-over ratios (SOR). The central $10 \mathrm{~mm}$ length of the rods were averaged, and circular regions-of-interest (ROIs) were drawn around each rod with diameters twice the diameters of the rods. The maximum value in each of the ROIs was measured and related to the average activity concentration of the uniform region to determine the recovery coefficients.

\section{RESULTS}

Figure 2 depicts representative slices of the reconstructed images. Visually, the image quality of the motion scan is comparable to the static scan.
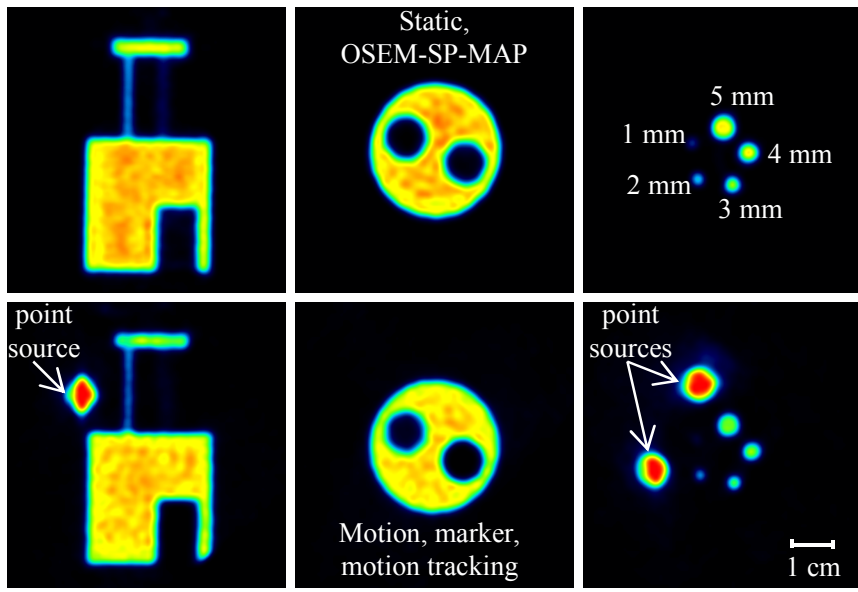

Fig. 2. Reconstructed images of the static and motion scans.

Table 1 shows the results of the uniformity test. In the static scan, the activity in the homogeneous region is overestimated by $27 \%$. This effect comes from the difference in size between the calibration phantom $\left(a^{68} \mathrm{Ge}\right.$ cylinder of $6 \mathrm{~cm}$ diameter $)$ and the smaller NEMA-phantom which is placed in the center of the FOV. In the motion scan, which is based on the same calibration, the activity is overestimated by only $14 \%$. Uniformity expressed as \%SD is $5.4 \%$ for the static scan, and $5.8 \%$ for the motion scan.

Spill-over ratios are summarized in Table 2. All ratios show very small values, showing an efficient scatter correction for both the static as well as the motion scan.

TABLE I. UNIFORMITY TEST FOR THE STATIC AND THE MOTION SCANS

\begin{tabular}{lllll} 
& $\begin{array}{l}\text { VOI Mean } \\
{[\mathrm{kBq} / \mathrm{cc}]}\end{array}$ & $\begin{array}{l}\text { Max } \\
{[\mathrm{kBq} / \mathrm{cc}]}\end{array}$ & $\begin{array}{l}\text { Min } \\
{[\mathrm{kBq} / \mathrm{cc}]}\end{array}$ & $\%$ \%SD \\
\hline Static, OSEM-SP-MAP & 208 & 262 & 169 & 5.4 \\
Motion, motion tracking & 146 & 178 & 113 & 5.8 \\
\hline
\end{tabular}

TABLE II. SPILL-OVER RATIOS (SOR) AS A MEASURE OF ACCURACY OF ATTENUATION AND SCATTER CORRECTIONS

\begin{tabular}{lllcr} 
& \multicolumn{3}{l}{ Water-filled cylinder } & Air-filled cylinder \\
& SOR & \%SD & SOR & \%SD \\
\hline Static, OSEM-SP-MAP & 0.010 & 80.3 & 0.023 & 47.7 \\
Motion, motion tracking & 0.043 & 15.4 & 0.002 & 59.6 \\
\hline
\end{tabular}

Recovery coefficients for the different rod sizes are shown in Figure 3. Even for the largest rods the recovery coefficient is far below 1 for the motion scan. This is due to the fact that the solid chamber around the rods contributes to attenuation but is not visible as activity contour (see Fig. 2). Attenuation correction therefore underperforms in this part of the phantom, which results in lower reconstructed average activity concentration in the rods, and therefore a recovery coefficient of less than 1. However, the flat shape of the curves for rod 
sizes above $3 \mathrm{~mm}$ in both the static and the motion scan as well as the visual impression (Fig. 2) indicate that the resolution is comparable.

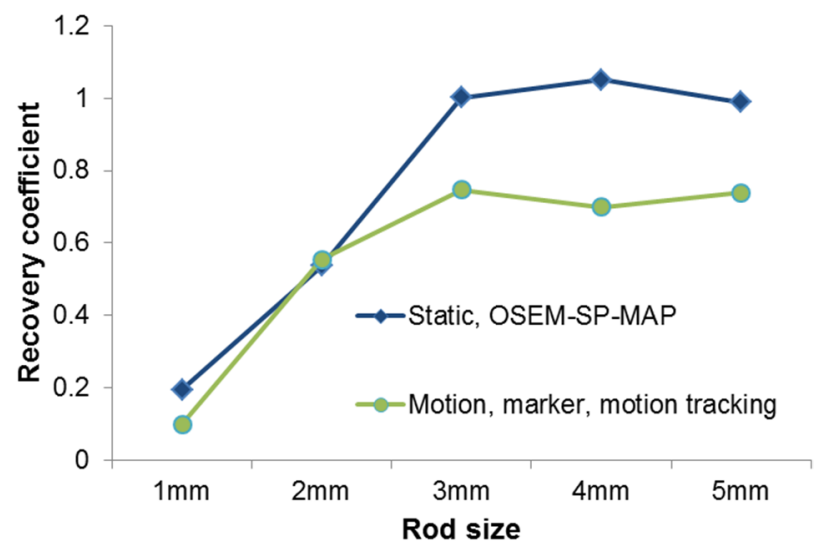

Fig. 3. Recovery coefficients for static and motion scans. The recovery coefficient $<1$ for the larger rods is due to insufficient attenuation correction for the special case of a PMMA phantom (see text).

\section{DISCUSSION AND CONCLUSION}

For the reconstruction of the motion scan, an approximate $\mu$-map is calculated from the activity outline of the phantom. With the incorporation of an attenuation and scatter correction based on this $\mu$-map, absolute quantification and the spill over ratios in cold regions in the motion corrected scan are comparable to the static scan, as long as the phantom outline is visible as activity contour.

In animal studies where the tracer distributes well over the animal body, the outline of the body can easily be estimated. For the phantom, the activity outline does not reflect well the shape of the phantom since not all parts of the phantom are active. This is the reason for the underestimation of the activity concentration of the rods in the reconstruction of the motion scan, and should be considered in animal studies with radiotracers accumulating highly in only a few regions so that the outline of the body is not well reflected.

Attenuation and scatter correction are not straightforward for awake animal scans and will be a topic of future research. Apart from this open issue, the most important conclusion is that even extensive motion, including rotation and turning front to back and including positions of the phantom in regions of low sensitivity within the FOV, affects image quality and quantitation only marginally when using pointsource based motion correction, compared to the motion free scan.

\section{ACKNOWLEDGMENT}

We thank Alexandra Drechsel and Nadja Hermes for their support in preparing the point sources.

\section{REFERENCES}

[1] C. DeLorenzo et al., "In vivo ketamine-induced changes in $\left[{ }^{11} \mathrm{C}\right] \mathrm{ABP} 688$ binding to metabotropic glutamate receptor subtype 5," (in eng), Biological psychiatry, vol. 77, no. 3, pp. 266-275, 2015.
[2] A. K. O. Alstrup and D. F. Smith, "Anaesthesia for positron emission tomography scanning of animal brains," Laboratory Animals, vol. 47, no. 1, pp. 12-18, Jan 2013.

[3] A. Z. Kyme, V. W. Zhou, S. R. Meikle, C. Baldock, and R. R. Fulton, "Optimised Motion Tracking for Positron Emission Tomography Studies of Brain Function in Awake Rats," Plos One, vol. 6, no. 7, Jul 1 2011.

[4] V. W. Zhou, A. Z. Kyme, S. R. Meikle, and R. Fulton, "An eventdriven motion correction method for neurological PET studies of awake laboratory animals," Molecular Imaging and Biology, vol. 10, no. 6, pp. 315-324, Nov 2008

[5] A. Kyme et al., "Markerless Motion Tracking of Awake Animals in Positron Emission Tomography," IEEE Transactions on Medical Imaging, vol. 33, no. 11, pp. 2180-2190, Nov 2014.

[6] A. Miranda, S. Staelens, S. Stroobants, and J. Verhaeghe, "Fast and Accurate Rat Head Motion Tracking With Point Sources for Awake Brain PET," IEEE Transactions on Medical Imaging, vol. 36, no. 7, pp. 1573-1582, Jul 2017.

[7] Performance Measurements for Small Animal Positron Emission Tomographs (NEMA Standards Publication NU 4-2008). Rosslyn, VA: National Electrical Manufacturers Association (NEMA), 2008.

[8] A. Miranda et al., "PET imaging of freely moving interacting rats," Neuroimage, vol. 191, pp. 560-567, May 12019.

[9] G. Angelis et al., "Calculated attenuation correction for awake small animal brain PET studies," in 2013 IEEE Nuclear Science Symposium and Medical Imaging Conference (2013 NSS/MIC), 2013, pp. 1-4. 